\title{
Strategies for Continual Application of Marker-Assisted Selection in an Open Nucleus Population
}

\author{
A. Stella, M. M. Lohuis, ${ }^{1}$ G. Pagnacco, ${ }^{2}$ and G. B. Jansen \\ Department of Animal and Poultry Science, \\ University of Guelph, \\ Guelph, Ontario, Canada N1G 2W1
}

\section{ABSTRACT}

The objectives of this study were to develop and simulate the implementation of several strategies for repeated application of quantitative trait loci (QTL) detection and marker-assisted selection (MAS) and to compare the short-term and continual genetic responses. A finite locus model was simulated with 20 QTL randomly distributed across 30 chromosome. Three hundred markers were evenly spaced across the genome. Allelic effects were sampled from a double exponential distribution. A daughter design was used every generation to determine the marker alleles favorably associated to QTL alleles. The MAS was applied within family to young bulls, before progeny testing, as part of an open nucleus. Young bulls were selected using strategies based on 1) the single marker with greatest contrast (BEST1), 2) the sum of $n$ greatest contrasts (BEST $n), 3$ ) the best $n$ contrasts, limited to one per chromosome (LIM $n), 4)$ the sum of all contrasts exceeding a given threshold $n$ (THRES $n$ ), and 5 ) the sum of contrasts exceeding a threshold, but limited to one per chromosome (LIMT). The maximum progress was achieved by strategies that selected upon several markers flanking multiple QTL in each generation. When THRES was applied, the mean true breeding value (TBV) of selected bulls was increased by $11.98 \%$ (over conventional selection) versus $6.73 \%$ for BEST1 in the first generation. Applying a full genome scan in each generation allowed selection for different QTL across time. By selecting for multiple QTL over time, MAS maintained superiority over conventional selection for many generations.

(Key words: quantitative trait loci, marker-assisted selection, genetic response)

\footnotetext{
Received October 11, 2001.

Accepted February 19, 2002.

Corresponding author: A. Stella; e-mail: stella@itba.mi.cnr.it.

${ }^{1}$ Current address: Animal Agricultural Group, Monsanto 700 Chesterfield Pkwy North, Chesterfield, MO 63198.

${ }^{2}$ Current address: VSA, Univ. of Milan, via Celoria 10, $20133 \mathrm{Mi}-$ lano, Italy.
}

\begin{abstract}
Abbreviation key: BESTn = MAS based on the $n$ markers with the greatest contrasts, GEN5 = five generations, LIMn = MAS based on the best markers on n chromosomes, LIMT = LIMn, but using only markers that exceed a threshold, MAS = marker-assisted selection, NEW = new, unrelated base population, SINGLE = single daughter design, $\mathbf{T B V}=$ true breeding value, THRES = MAS based on all markers that exceed a given threshold.
\end{abstract}

\section{INTRODUCTION}

Numerous studies have demonstrated that markerassisted selection (MAS) is effective in increasing genetic response in the short term (Whittaker et al., 1995; Ruane and Colleau, 1996; Mackinnon and Georges, 1998; Spelman and Garrick, 1998). These studies have generally examined selection at a single locus across the population. However, when selection occurs, the variability of alleles at a QTL decreases from generation to generation. This decrease may be especially large at loci where direct MAS is applied (Stella et al., 2001). Advantages of MAS programs can, therefore, be sustained in the long term only if new QTL are continually discovered and selected. Retesting for QTL may also be necessary to account for recombination between the QTL and the markers (Zhang and Smith, 1993; Whittaker et al., 1995). These new QTL may be smaller than the original QTL, because the larger QTL are the most likely to be found early or moved toward fixation via the forces of conventional selection. Thus, the gains in later generations may be reduced because the maximum potential (allelic substitution effect) of successive QTL is likely to be smaller and the type I error of the QTL detection test is likely to be larger (Whittaker et al., 1995; Meuwissen and Goddard, 1996; Spelman and Van Arendonk, 1997; Villanueva et al., 1999).

The objective of this study was to develop and simulate several strategies for repeated application of QTL detection and MAS and compare the strategies for short-term and continual genetic response. The sustainability of genetic response over several genera- 


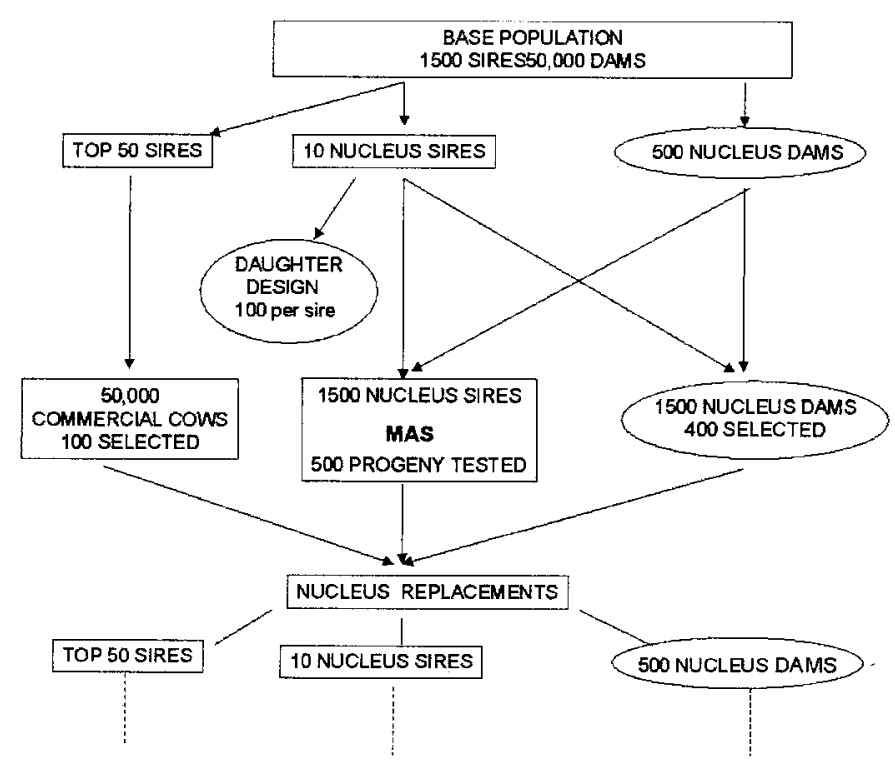

Figure 1. Population structure.

tions of QTL detection and MAS in a dairy nucleus herd was compared to genetic progress achieved with conventional selection. Sustainability was evaluated by the number of QTL exploited in later generations compared with early generations. Stochastic simulations were developed based on a finite locus model.

\section{MATERIALS AND METHODS}

The simulated population included an open nucleus and a commercial herd. Selection for a sex-limited trait was modeled. The MAS was applied based on a complete genome scan during each generation, using the bottom-up design by Mackinnon and Georges (1998). Associations between QTL and markers were identified based on genotypes and phenotypic records of progeny test daughters. This information was used for within-family selection of young bulls entering the progeny test program.

\section{Population}

The structure is shown in Figure 1. The base population consisted of 1500 and 50,000 unrelated and unselected males and females, respectively. To form the nucleus, the top 10 sires $(0.66 \%)$ and top 500 dams (1\%) were selected from the base population according to their EBV. For all individuals, EBV were simulated by adding random, normally distributed, prediction errors to the true breeding value (TBV), using the approach of Spelman and Garrick (1998):

$$
\begin{aligned}
\mathrm{EBV}_{\mathrm{i}}=\mathrm{r}_{\mathrm{TI}}^{2}\left(\mathrm{BV}_{\mathrm{i}}-\mathrm{MBV}\right)+\mathrm{z}_{\mathrm{i}} * \mathrm{~V}\left(\mathrm { V } _ { \mathrm { p } } \left(\mathrm{~h}^{2} \mathrm{r}_{\mathrm{TI}}^{2}\right.\right. & \left.\left.-\mathrm{h}^{2} \mathrm{r}_{\mathrm{TI}}^{4}\right)+\mathrm{MBV}\right)
\end{aligned}
$$

where, EBV was estimated breeding value for animal $\mathrm{i}, \mathrm{r}_{\mathrm{TI}}^{2}$ was the squared accuracy of selection, $\mathrm{BV}_{\mathrm{i}}$ was the true breeding value, MBV was the population mean breeding value in the current generation, $z_{i}$ was a standard normal deviate, and $V_{p}$ was the phenotypic variance. The standard deviation of the prediction error was varied by altering $\mathrm{r}_{\mathrm{TI}}^{2}$ according to sex and generation to reflect the amount of information typically available for prediction of BV. The EBV of the sires were assumed to have accuracies corresponding to a progeny test with 100 daughters. Dams for the nucleus were selected based on EBV with accuracy corresponding to one lactation record. Each of the ten sires was mated randomly to 50 nucleus dams to generate full-sib families of three males and three females. Within each family, the best male was selected based on his genotype, considering results of a daughter design conducted on each of the ten sires. All $1500 \mathrm{fe}-$ males produced were eligible for selection on their EBV, which had accuracies comparable to one completed lactation. No additional fixed effects (such as management group, age, or parity) were generated. Among these females, the top 400 were selected as part the next generation of dams. In addition, 100 dams were randomly selected from the top $2 \%$ of a commercial population. The commercial population was comprised of 50,000 cows that were sired by the top 50 bulls in the previous generation. These cows had one gamete from one of the sires, and the other gamete was generated based on allelic frequencies in the commercial population. The allelic frequencies in the commercial population were computed in each generation as the average of previous generation and the average of the 50 selected bulls. For the base scenario, five generations were simulated.

\section{Genetic Model}

The TBV were produced assuming a finite locus model for which all the allelic effects were strictly additive. The genetic model was based on the general approach of Mackinnon and Georges (1998). The parameters used to simulate the base situation are in Table 1. Several comparisons were made for certain scenarios generated with alternative parameters. In the base situation, all the genetic variability in the trait of interest was explained by 20 loci, each with five alleles. Mackinnon and Georges (1998) also considered QTL with multiple alleles. Multiple alleles have been reported for several genes, including blood groups, milk protein genes, and loci in the major histocompatability 
Table 1. Simulation parameters.

\begin{tabular}{lcl}
\hline Parameter & Base scenario & Alternatives \\
\hline Heritability & 0.30 & $0.10,0.50$ \\
Number of QTL & 20 & 50 \\
Recombination rate between markers $^{1}$ & 0.10 & $0.05,0.15$ \\
MAS strategy $^{1}$ & BEST1 & BEST $n$, LIM $n$, THRES $n$, LIMT, SINGLE \\
\hline
\end{tabular}

${ }^{1} \mathrm{BEST} n=$ marker assisted selection based on $n$ highest marker contrasts, LIM $n=$ selection on best single markers on $n$ chromosomes, THRES $n=$ selection on all markers with contrasts greater than threshold $n$, LIMT = selection on markers that exceed a threshold, but limited to one per chromosome, SINGLE = selection based on results from a single QTL detection study, in the first generation.

complex. Different QTL effects and positions were randomly assigned in each replicate. Allelic effects were simulated using a double exponential distribution. Compared to the normal distribution, the double exponential distribution is more sharply peaked, yielding relatively fewer intermediate allelic effects and slightly more large ones (Figure 2). The initial QTL allele frequencies were uniformly distributed and standardized to sum to 1.0. The phenotypic variance was 400 . In the base scenario, heritability was 0.30 . The QTL were randomly distributed across the genome, with no upper limit placed on the number of QTL per chromosome.

Thirty chromosomes of $90 \mathrm{cM}$ each were simulated. Three hundred codominant markers, with six alleles each, were distributed across the 30 chromosomes, evenly spaced, and separated by a recombination rate $(\theta)$ of 0.10 .

\section{Marker-Assisted Selection}

A daughter design was used every generation to determine which marker alleles were associated with the

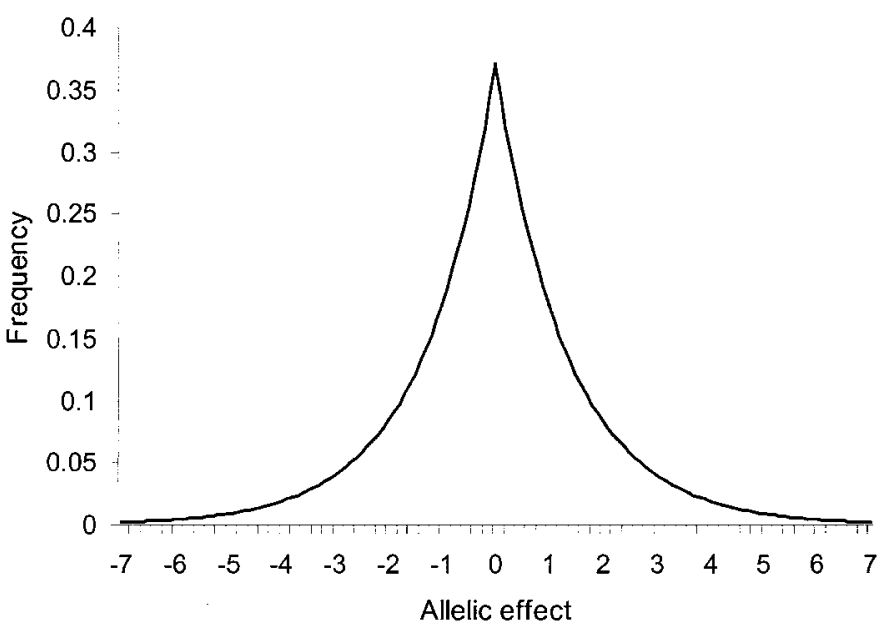

Figure 2. Double exponential distribution of allelic effects used in this study. favorable QTL alleles. Each sire had 100 daughters from the commercial population. Each daughter had a single lactation record and a complete marker genotype. Marker contrasts were calculated for all heterozygous markers within sire. All daughters that were informative at a given locus were used to calculate the contrast, ignoring the genotype of the dams. The contrast was simply the difference in the mean of EBV of daughters carrying the alternative sire alleles. These EBV were based on one lactation record. Contrasts were then divided by their standard errors to account for differences in the number of informative daughters across loci.

Marker-assisted selection was applied to select the best sons from among full-sib families of three males. Selection was based on one or more of the markers with the highest contrasts. If all the sons were uninformative or carried the same sire allele, then selection was random.

The efficiency of QTL detection experiments and MAS varies depending on the genetic variance (Lande and Thompson, 1990; Ruane and Colleau, 1996; Meuwissen and Goddard, 1996), so two alternate levels of heritability were also considered $(0.10$ and 0.50 , Table 1). Also, the effect of the map density was examined. In addition to the base scenario, responses to selection were examined when using a relatively $\operatorname{FINE}(\theta=0.05)$ or COARSE $(\theta=0.15)$ map of genetic markers. For these alternative maps, the number of markers was changed and the length of the genome remained constant.

\section{Selection Strategies}

Five different selection strategies were examined (Table 1). In the first approach (BEST1), which served as the basis for most comparisons, the selection was based on the single best marker contrast for each sire, where the "best" marker was the marker with the greatest contrast (absolute value). The selected marker could be different for each sire. The second strategy selected the young bull based on the best $n$ marker loci, where $n$ was equal to 3, 5, and 10 (BEST3, 
BEST5, and BEST10, respectively). For each son, an index was calculated upon which to base the selection:

$$
I_{i j}=\sum_{k=1}^{n}(-1)^{m} * C_{i k},
$$

where $i$ is the sire number, $j$ is the son number, $k$ is the locus number, $n$ is 3,5 , or $10, C_{i k}$ is the contrast for sire $i$ at locus $k$, and $m$ is and indicator variable with value 0 or 1 if the first or second sire allele was inherited, respectively. This index differed for each son depending on which sire alleles were inherited.

The third strategy was a variation of the second. This approach first identified the marker with the highest contrast for each chromosome and then selected bulls based on an index (equation 2) for the best $n$ of these markers. The limit of a single marker per chromosome was designed to prevent selection on multiple markers linked to the same QTL. Two levels of $n$ were used, 3 and 5 markers (LIM3 and LIM5, respectively).

The fourth strategy (THRES) involved considering all the markers for which the standardized contrast exceeded a given threshold. For the base situation, the threshold was set somewhat arbitrarily at 1.96, consistent $\alpha=5 \%$ significance test (THRES5) for a single experiment, although many markers were tested. Because of the arbitrary nature of this threshold and because multiple tests were made, for comparison, the simulation was repeated by using lower (THRES10) and higher (THRES1) to reflect $\alpha=10 \%$ and $\alpha=1 \%$, respectively.

The fifth strategy, LIMT, combined aspects of LIM and THRES. First, the marker with the greatest contrast was identified for each chromosome. Then selection was based on all of these 30 markers with contrasts that exceeded the threshold 1.96. This threshold was based on a $95 \%$ confidence interval with a normal distribution. Following this convention, thresholds of 1.65 and 2.58 were used for THRES10 and THRES1, respectively.

The final strategy (SINGLE) evaluated the feasibility of applying the results of a single daughter design in the first generation to making selection decisions in subsequent generations without repeating the genome scan. Markers were traced from the initial sires of sons to their offspring, and MAS was applied to choose sons of informative sires.

No specific effort was made to finely map and characterize the QTL, but the QTL were assumed to be nearest to the markers with the greatest contrast. Because selection was based on an index of contrasts [Equation 2], the markers most likely to be near QTL implicitly received the greatest weight in the index.
Generational and cumulative responses to MAS were compared to response to conventional selection (random within-family selection). The extra gain per generation from MAS was the difference between the means of TBV of bulls selected using markers and bulls selected randomly. This quantity was expressed as a percentage by dividing by the gain achieved through random selection.

Many of the past theoretical studies on MAS have assumed a single QTL (Ruane and Colleau, 1996; Spelman and Garrick, 1998), clusters of QTL (Meuwissen and Van Arendonk, 1992; Meuwissen and Goddard, 1996) or few QTL (Spelman and Bovenhuis, 1998; Schulman et al., 1999), with previous knowledge of the position of the QTL relative to flanking markers. Thus, in these studies, the QTL being selected was predetermined. In contrast, the present study assumed no previous knowledge of QTL location. Therefore, several analyses were performed to investigate which markers were used in selection decisions, their location relative to the QTL, and the size of the QTL, in terms of genetic variance. To do this, the marker upon which selection was applied was monitored for each sire. The location of the marker relative to the nearest QTL was observed. The proportion of times that a QTL was adjacent to the marker, within three marker intervals (i.e., approximately $30 \mathrm{cM}$ ) or simply on the same chromosome was recorded. In addition, the genetic variance (in the commercial population) contributed by the marked QTL was recorded and all QTL were ranked in terms of their genetic variance in the commercial population.

Some previous studies (e.g., Schulman et al., 1999) have shown that MAS induces negative linkage disequilibrium among QTL greater than that expected to occur as a result of conventional selection. Negative linkage disequilibrium occurs when a negative covariance exists between the selected locus and the remaining loci. This disequilibrium has the potential to reduce genetic gain from MAS and possibly diminish the power of experiments designed to detect QTL. To test for evidence of increased disequilibrium (resulting in decreased power for QTL detection) due to MAS, we compared the mean value of contrasts following five generations of MAS versus five generations of conventional selection. However, changes in the relative magnitude in the contrasts between MAS and conventional selection could also be the result of differences in allelic frequencies and decreased genetic variance due to increased selection response from MAS. To account for differences in the contrasts due to changes in allelic frequencies, the allelic frequencies for both MAS and conventional schemes were recorded after five generations (GEN5) and then used as initial gene 
frequencies to establish a new, unrelated base population (NEW). Then, marker contrasts were reestimated in this NEW population. The difference between maximum marker contrasts from the GEN5 and NEW were then compared for MAS and conventional selection. A significant difference between the magnitude of contrasts from GEN5 and NEW populations was assumed to indicate a difference in disequilibrium.

To compare long-term responses for MAS and conventional selection, the simulation was allowed to continue for many $(\geq 30)$ generations until the genetic variance was nearly exhausted $(<0.1 \%$ of the original variance) and cumulative response to MAS was compared to conventional selection. Much uncertainty and controversy exists regarding the true number of genes affecting quantitative traits (Lande, 1981; Zeng, 1993). Therefore, for this comparison, a situation in which the trait was controlled by 50 QTL with five alleles each (Hayes and Goddard, 2000) was also simulated.

\section{RESULTS AND DISCUSSION}

The following results pertain to the base set of parameters outlined in Table 1 unless stated otherwise.

The genetic variance accounted for by each QTL was monitored. With 20 QTL, each QTL was expected (on average) to account for $5 \%$ of the total genetic variance within a particular replicate. However, the size of effects varied for each QTL and $13.1 \%$ of the individual QTL accounted for at least $10 \%$ of the total genetic variance in their respective replicate. Also, $2.3 \%$ of the QTL accounted for at least $20 \%$ of the variance in their replicate, and 0.5 and $0.1 \%$ of the individual QTL accounted for at least 30 and $40 \%$, respectively. On average, the maximum difference between the highest and lowest allelic effect for any QTL was 9.90, which corresponded to approximately 0.9 genetic SD or 0.5 phenotypic SD. Previous analyses of field data (Ashwell et al., 1997; Georges et al., 1995) have uncovered QTL with estimated effects of this size or greater.

Table 2 shows the percentage increase above random selection in the average TBV of the chosen progeny test bulls for each of the five MAS approaches. Each percentage point increase corresponded to a difference of approximately 0.028 genetic SD in the difference of mean TBV between bulls selected randomly and by MAS. The percentage increase ranged from $6.73(0.19$ genetic SD) for the BEST1 to 11.98 (0.33 genetic SD) for THRES5, in the first generation. Gains were roughly halved by GEN5.

Cumulative gains in the mean TBV of the commercial population are also presented (Table 2), and they followed the same pattern. Cumulative gains in the commercial population (ranging from 1.68 to $2.77 \%$ ) were less than the average advantage per generation for young bulls because selection of young bulls is only one of the factors that affect genetic progress in the commercial population and MAS was applied only in this pathway.

One can consider BEST1 and BEST3 as applications of THRES with much higher thresholds. On average, 19 marker locations exceeded the 1.96 critical value for THRES5 (compared to 1 and 3 for BEST 1 and BEST3). Clearly, some of the potential gain from MAS was lost when a too stringent critical value was applied. Basing selection decisions on a fixed threshold rather than on a fixed number of loci allows many potential loci on several chromosomes to be considered, if statistical evidence supports the segregation of numerous loci. In addition, the threshold method easily allows for selection based on an interval of markers surrounding the same QTL. This helps to account for imprecision in the location of the QTL with respect to the markers (in contrast, BEST1 assumes that the QTL is in the interval adjacent to the marker), therefore increasing response to MAS (Stella et al. 2001). Another potential advantage for the threshold was that it could allow for variability among the sires in the numbers of marker contrasts used in selection. With the THRES5, sires ranged from 4 to $39(\mathrm{SD}=6.7)$ in the number of marker contrasts that exceeded the threshold, which indicates variability among sires in the number of segregating QTL with widely different allelic effects. When BEST10 was applied, cumulative gain was $2.80 \%$, which exceeded (although not significantly) the gain from THRES5. Therefore, the advantage of THRES5 over the other approaches was more a function of selection on more loci than of allowing variability among sires in the number of markers that contributed to the selection criterion.

Some efficacy was lost by applying a very liberal threshold because of excessive type I errors. For this simulated population, the optimal threshold was within the range corresponding to a comparison wise $\alpha$ of between 1 and 5\%. When applying the higher threshold $(\alpha=1 \%)$, the cumulative genetic gain over five generations was $2.84 \%$, which was not significantly greater than the response of $2.77 \%$ achieved when the threshold was $\alpha=5 \%$. When the lower threshold ( $\alpha=10 \%)$ was applied, cumulative genetic gain was significantly $(P<0.01)$ decreased, at $2.52 \%$.

Response to MAS was decreased, relative to THRES5, when applying the LIM3, LIM5, and LIMT approaches, which restricted selection to a single marker per chromosome. Although these methods outperformed BEST1 because they allow for selection on multiple QTL, by restricting selection to a single 
Table 2. Average (SD) increase ${ }^{1}$ in mean true breeding value of the selected group of young bulls when marker-assisted selection (MAS) is applied within family and cumulative gain (relative to conventional selection) in the commercial populations after five generations of selection.

\begin{tabular}{|c|c|c|c|c|c|}
\hline \multirow[b]{2}{*}{ Generation } & \multicolumn{5}{|c|}{ MAS method ${ }^{2}$} \\
\hline & BEST1 & BEST3 & THRES5 & LIM3 & LIMT \\
\hline & & & $\%$ & & \\
\hline 1 & $6.73^{\mathrm{a}}(2.94)$ & $10.14^{\mathrm{b}}(3.34)$ & $11.98^{\mathrm{c}}(3.35)$ & $9.72^{\mathrm{d}}(3.33)$ & $9.55^{\mathrm{d}}(2.99)$ \\
\hline 2 & $5.34^{\mathrm{a}}(2.94)$ & $8.32^{\mathrm{b}}(3.46)$ & $9.85^{\mathrm{c}}(3.47)$ & $7.91^{\mathrm{d}}(3.45)$ & $7.89^{\mathrm{d}}(3.23)$ \\
\hline 3 & $4.82^{\mathrm{a}}(2.85)$ & $7.44^{\mathrm{b}}(3.33)$ & $8.62^{\mathrm{c}}(3.32)$ & $6.97^{\mathrm{d}}(3.48)$ & $7.05^{\mathrm{d}}(3.07)$ \\
\hline 4 & $4.16^{\mathrm{a}}(2.95)$ & $6.25^{\mathrm{b}}(3.30)$ & $7.04^{\mathrm{c}}(3.40)$ & $5.94^{\text {bd }}(3.30)$ & $5.69^{\mathrm{d}}(2.91)$ \\
\hline 5 & $3.43^{\mathrm{a}}(2.81)$ & $5.20^{\mathrm{b}}(3.41)$ & $5.79^{\mathrm{c}}(3.41)$ & $4.81^{\mathrm{b}}(3.20)$ & $4.63^{\mathrm{b}}(2.80)$ \\
\hline Cumulative & $1.68^{\mathrm{a}}(0.78)$ & $2.31^{\mathrm{b}}(0.82)$ & $2.77^{\mathrm{c}}(0.82)$ & $2.29^{b}(0.81)$ & $2.34^{\mathrm{b}}(0.80)$ \\
\hline
\end{tabular}

marker per chromosome, some precision was lost when the underlying QTL was not in an interval adjacent to the selected marker. A selected son could share the sire's haplotype at the selected markers but not the desired QTL allele, if recombination occurred. No significant differences in selection response were observed when considering 3 versus 5 loci (LIM3 versus LIM5).

For all approaches, the advantages of MAS decreased with each generation. These decreases occurred because selection decreased the amount of genetic variability in the population and, therefore, the expected difference between sire alleles at the QTL. This loss of variability not only decreased the potential gain that could be achieved, but also decreased the accuracy of the estimates of associations between markers and QTL. The number of markers exceeding the given thresholds also decreased each generation.

Estimates of the benefits of MAS from this study fall within the range of previous estimates for dairy populations, but estimated benefits of MAS have varied from study to study depending on how MAS was applied. When applied for young bull selection, Mackinnon and Georges (1998) reported an increase in the genetic level of selected bulls of $10 \%$, and Spelman and Garrick (1998) reported gains of up to $9 \%$ higher when MAS was applied. Gains due to MAS could have been increased if MAS had been used for both nucleus bulls and heifers and if genotypic information from dams had been considered. Ruane and Colleau (1996) obtained an increase in genetic progress of up to $15 \%$ when MAS was applied in a more comprehensive manner.

Results for the model with 50 QTL were similar to those for 20 QTL in that the different strategies ranked the same in efficacy. However, gains over random se- lection were relatively smaller in earlier generations but were maintained at a higher level in later generations. For example, using the BEST1 procedure, the gain over random selection was only $5.12 \%$ (vs. $6.73 \%$ with 20 QTL) in generation 1 but $4.36 \%$ (3.43\%) in generation 5 . This trend result occurred because effects of individual QTL were greater with 20 QTL than with 50 QTL (because the same amount of variability was shared by fewer QTL). Thus, with 20 QTL, the largest QTL were more accurately identified and selected for in early generations, leaving less genetic variance (and opportunity for selection response) in later generations.

The risk associated with the various strategies can be evaluated by comparing the mean advantage in TBV of selected bulls with the standard deviation of this advantage (Table 2). For example, in generation 1 , the advantage of the BEST1 strategy was $6.73 \%$ with a $\mathrm{SD}=2.94 \%$. Thus, the TBV of bulls selected by BEST1 exceeded that of the randomly selected bulls by an average of $2.28 \mathrm{SD}$. Assuming that the advantage was normally distributed, the randomly selected young bulls were expected to be equal or superior to the MAS bulls in only about $1 \%$ of the replicates. Not surprisingly, the more successful strategies were even less risky, as the means in the advantages of these strategies in TBV for generation 1 were all in the range of 3.0 SD, meaning that the TBV of the randomly selected bulls would be expected to meet or exceed the TBV of MAS bulls in fewer than $0.15 \%$ of replicates.

As expected, the risk increased as more generations of MAS were practiced and the advantage of the MAS bulls decreased. In GEN5, the TBV of THRES5 bulls was only 1.70 SD greater than randomly selected bulls on the average, so the randomly selected bulls were 
Table 3. Relative frequency of finding the largest marker contrast near a QTL and the expected values for a marker selected at random.

\begin{tabular}{llll}
\hline & \multicolumn{3}{c}{ Position of the best contrast } \\
\cline { 2 - 4 } Generation & $\begin{array}{l}\text { Within } \\
\pm 10 \mathrm{cM}\end{array}$ & $\begin{array}{l}\text { Within } \\
\pm 30 \mathrm{cM}\end{array}$ & $\begin{array}{l}\text { Whole } \\
\text { chromosome }\end{array}$ \\
\hline & & & \\
1 & 56.9 & 78.7 & 87.2 \\
2 & 47.5 & 69.9 & 80.6 \\
3 & 43.2 & 65.6 & 76.9 \\
4 & 37.9 & 59.6 & 72.9 \\
5 & 33.8 & 54.9 & 69.0 \\
Random & 12.5 & 29.9 & 49.2 \\
\hline
\end{tabular}

superior to the MAS bulls about $4.5 \%$ of the time. This level of risk was still rather low.

The cumulative response in the commercial population after five generations followed similar patterns. When BEST1 was practiced, the mean TBV of the commercial population was approximately $2.15 \mathrm{SD}$ above the mean when only conventional selection was practiced ( $1.58 \%$ risk). By practicing THRES5, this risk was $<0.05 \%$.

Table 3 gives, for each generation, the frequency for which the marker with the highest contrast was located near a QTL, for the BEST1 strategy. Clearly, the probability that a QTL existed nearby the marker with the highest contrast decreased over generations. For example, in the first generation, there was a $60 \%$ probability that a QTL was located in the interval between the selected marker and the next marker on the chromosome. This dropped by almost half (to $33.8 \%$ ) in generation 5. Nevertheless, MAS was still effective in the fifth generation because this percentage was still 3 times greater than probability expected by choosing a marker at random. In addition, about $55 \%$ of the time a QTL was within $30 \mathrm{cM}$ from the marker with the best contrast and $69 \%$ of the time on the same chromosome. This result stresses the importance of considering multiple markers by using one of the other methods such as THRES. Approximately $12 \%$ of the time no QTL was found on the same chromosome as the selected marker, even in the first generation. This percentage increased to $31 \%$ in the fifth generation, as the largest QTL approached fixation for the best alleles and the segregating QTL became more difficult to identify.

Although the marker with the largest contrast was usually near a QTL, this QTL was rarely the most important locus, as measured by the amount of genetic variance (in the commercial population) accounted for by the QTL (Table 4). For each replicate and generation, each QTL was ranked based on its genetic variance in the commercial population. For each sire, we determined the QTL that was being selected by the marker with the greatest contrast. Only $10.1 \%$ of the times did the selected QTL have the most variance in the commercial population.

On average, selection was for the seventh most variable QTL in generation 1 and this value decreased with increasing generations (Table 4). When using an additive model, the variance accounted for by a QTL is a function of allelic frequencies and substitution effects (Falconer and McKay, 1996). With large substitution effects, conventional selection would favor animals that are homozygous for the most favorable allele. Therefore, even if a QTL had the greatest genetic variance in the commercial population, many of the nucleus sires may have been homozygous for the most favorable allele (Table 4). If so, then markers near this QTL would not show a large contrast. Spelman and Garrick (1998) demonstrated, under similar conditions of selection intensity but using a single QTL, that the average contrast decreased by $25 \%$ in two generations of selection. For each QTL, the correlation for that QTL was calculated between the genetic variance contributed by that QTL and the proportion of sires that were homozygous for that QTL. We also recorded the proportion of sires that were homozygous at the QTL that contributed the most genetic variance. The correlation between QTL variance and proportion

Table 4. Average rank in genetic variance ${ }^{1}$ of the QTL selected, the average percentage of sires that were homozygous for the QTL that contributed the greatest genetic variance and the correlation $(\mathrm{r})$ between the rank of the genetic variance contributed by each QTL with the proportion of sires that were homozygous for that QTL.

\begin{tabular}{llll}
\hline Generation & Rank $^{2}$ & $\begin{array}{l}\text { Homozygous } \\
\text { sires }\end{array}$ & $\mathrm{r}$ \\
\hline & & $(\%)$ & \\
1 & 7.16 & 30.6 & 0.23 \\
2 & 9.06 & 37.7 & 0.38 \\
3 & 10.05 & 44.2 & 0.47 \\
4 & 11.00 & 52.3 & 0.51 \\
5 & 11.91 & 60.3 & 0.53 \\
\hline
\end{tabular}

${ }^{1}$ In to the commercial population.

${ }^{2}$ Out of 20 . 
Table 5. Effect of marker density on the increase ${ }^{1}$ in mean true breeding value of the selected group of young bulls when marker assisted selection is applied within family.

\begin{tabular}{llll}
\hline & \multicolumn{3}{c}{ Marker density $^{2}$} \\
\cline { 2 - 4 } Generation & COARSE & BEST1 & FINE \\
\hline 1 & 6.28 & 6.73 & 6.96 \\
2 & 4.85 & 5.34 & 5.60 \\
3 & 4.25 & 4.82 & 5.05 \\
4 & 3.63 & 4.16 & 4.37 \\
5 & 3.08 & 3.43 & 3.58 \\
\hline
\end{tabular}

${ }^{1}$ Expressed as a percentage of the genetic response with conventional selection.

${ }^{2}$ Recomination rate $=0.15,0.10$, and 0.05 , for COARSE, BEST1, and FINE, respectively.

of homozygous sires was positive and significant $(P<$ 0.0001 ), ranging from 0.23 in generation 1 to $>0.5$ after generation 3 (Table 4). The proportion of sires homozygous at the most variable QTL was 0.3 in first generation (expected $\sim 0.25$ in the absence of selection) and more than 0.6 in generation 5 .

Although QTL detection experiments have been shown to be most powerful when applied across family (Spelman et al., 1999), these results underscore the importance of considering results within family when subsequently applying MAS. Not only must transmission studies within family be used to determine the phase of the association between marker and QTL (Weller et al., 1990), but also to determine whether each family is actually segregating. These results suggest that as QTL variance (and thus the power for detection) increases, the probability that the QTL will be segregating within highly selected animals is decreased.

No difference was observed in the relative effectiveness of MAS with respect to the true heritability of the trait. For all three heritability levels $(0.10,0.30$, 0.50 ), the average TBV for young bulls chosen with MAS in the first generation was $6.73 \%$ greater than of those bulls selected randomly. These percentages varied slightly in later generations, but not significantly. With a lower heritability, the marker contrasts were estimated less accurately, but this factor seemed to be balanced by the fact that sires were less likely to be homozygous for the most variable QTL. These results differed from other studies (Ruane and Colleau, 1996; Stella et al., 2001), but those studies considered only a single QTL. Moreau et al. (1998) showed that the response to MAS decreased for very low heritabilities $(<0.15)$. They estimated the existence of an optimal heritability around 0.15 to 0.20 , varying slightly depending on the percentage of genetic variance associated with the markers.

The effect of marker density on efficiency of MAS was also examined (Table 5). As expected, finer map- ping yielded a superior group of progeny test bulls. However, the marginal advantage of increasing the marker density decreased as the density increased. For, example, when the COARSE mapping strategy was applied in generation 1, the average TBV of selected young bulls exceeded the EBV of randomly selected bulls by $6.28 \%$ (Table 5). This gain was increased by $7.2 \%$ (to $6.73 \%$ for BEST1) by increasing the marker density by $67 \%$ (from 6 to 10 markers per chromosome). Increasing the number of markers per chromosome by an additional $80 \%$ (from 10 to 18 with FINE) resulted in an increase of only $3.4 \%$ (to $6.96 \%$ ). In fact, when cumulative gain was considered over five generations, the difference between using the base (BEST1) and FINE map was not significant $(P>0.10)$. The reason to place several markers on a chromosome is to use recombination events between markers to help locate the QTL more precisely. Spelman and Bovenhuis (1998) showed that, for this reason, having smaller marker brackets increased the response to MAS. In our study, diminishing returns with finer maps occurred because, as maps become more dense, the number of daughters (or sons) with a recombination between adjacent markers decreases.

Some breeding companies may be interested in trying to apply the SINGLE approach because the QTL detection is only performed once and, therefore, costs decrease greatly. However, the benefits of applying MAS also decreased greatly. In the second generation of MAS, the average TBV of young bulls was only $1.27 \%$ greater than random within-family selection, and the advantage was nearly negligible in later generations. The reason for this steep decline in the advantage of MAS is clear. If within-family MAS is effective, nearly all surviving members of a given sire family will have inherited the best alleles at the loci upon which MAS was applied. Thus, little additional benefit can be obtained by considering this locus in future generations within that family.

Means of the maximum contrasts (absolute value) following five generations of conventional selection and MAS are in Table 6 . The means of maximum contrasts when the resulting allelic frequencies were used to establish new populations (NEW) are also presented. The maximum contrast following MAS (3.18) was significantly $(P<0.0001)$ but only slightly lower than after the same period of random within-family selection of progeny test bulls (3.26). No difference was observed in the maximum contrasts for NEW populations established with the resulting allelic frequencies, so the difference between GEN5 and NEW contrasts was also greater $(P<0.0001)$ for MAS than conventional selection. This difference was likely due to increased negative linkage disequilibrium among QTL 
Table 6. Mean of maximum contrasts ${ }^{1}$ following five generations (GEN5) of conventional and marker-assisted selection (MAS) and in unrelated populations with the same allelic frequencies (NEW).

\begin{tabular}{lcr}
\hline & \multicolumn{2}{c}{ Selection scheme } \\
\cline { 2 - 3 } Population & Conventional & MAS \\
\hline GEN5 & $3.26^{\mathrm{a}}$ & $3.18^{\mathrm{b}}$ \\
NEW & $3.46^{\mathrm{a}}$ & $3.46^{\mathrm{a}}$ \\
Difference ${ }^{2}$ & $-0.20^{\mathrm{a}}$ & $-0.28^{\mathrm{b}}$ \\
\hline${ }^{\mathrm{a}, \mathrm{b}}$ Values with different superscripts in each row are significantly \\
different $(P<0.0001)$.
\end{tabular}

when MAS was applied. Contrasts were greater in NEW versus GEN5 populations for both selection schemes. Increased genetic relationships among animals in the commercial population after five generations of selection were likely responsible for much of this difference, but should not have greatly affected the difference between MAS and conventional selection in GEN5 because MAS was applied only within families.

Previous studies have demonstrated inferior longterm genetic response to MAS compared with conventional selection (Gibson, 1994; Villanueva et al., 1999). That phenomenon was not observed in our study for either 20 or 50 QTL models. Cumulative response with MAS was consistently greater than with random selection within full-sib family, even after selection had continued to the point where genetic variance was reduced to less then $0.01 \%$ of the initial variance. Our study differed from the previous, however, in several features. First, selection strategies differed. In our study, MAS was applied only to choose progeny test bulls from within families. In contrast, Gibson (1994) and Villanueva et al. (1999) practiced population wide selection based on an index of the marked gene and the remaining polygenes. Also, previous studies simulated continued MAS on a single locus, rather than a varying array of loci as was the case in the present study. Applying MAS on many loci may be a more robust approach that helps prevent drift from fixing some loci at nonfavorable alleles.

\section{CONCLUSIONS}

Applying full genome scans in each generation allowed for selection on different QTL across time. Within a given family, repeated selection for the same marker locus over multiple generations was of little value because of loss of information and decreased genetic variability from previous selection. Maximum progress was achieved by selecting in each generation upon several sets of markers neighboring multiple potential QTL, rather than only on markers near the single most likely QTL. The markers selected in each family should be based on a significant threshold rather than on arbitrary limitations on number of markers per chromosome or family.

The cost of the simulated MAS program would likely be prohibitive with current genotyping technology. Therefore, perhaps some combination of repeated scans on targeted areas of the genome and application of previously obtained results may be more efficient for breeding companies. Future technologies may, however, greatly decrease the cost of repeated full genome scans.

Within family MAS was rarely applied to the most important QTL in the commercial population because sires of sons were often homozygous for the best alleles at these loci. When MAS was practiced on different QTL in different generations, cumulative response with MAS maintained an advantage over response from conventional selection.

\section{REFERENCES}

Ashwell, M. S., Y. Da, P. M. VanRaden, C. E. Rexroad, Jr., and R. H. Miller. 1997. Detection of loci affecting milk production and health traits in an elite US Holstein population using microsatellite markers. Anim. Genet. 28:216-222.

Falconer, D. S., and T. F. C. Mackay. 1996. Introduction to Quantitative Genetics. 3rd ed. Longman, Essex, UK.

Georges, M., D. Nielsen, M. Mackinnon, A. Mishra, R. Okimoto, A. T. Pasquino, L. S. Sargeant, A. Sorensen, M. R. Steele, X. Zhao, J. E. Womack, and I. Hoeschele. 1995. Mapping quantitative trait loci controlling milk production in dairy cattle by exploiting progeny testing. Genetics 139:907-920.

Gibson, J. P. 1994. Short-term gain at the expense of long-term response with selection of identified loci. Proc. 5th World Cong. Genet. Appl. Livest. Prod. 21:201-204.

Hayes B., and M. E. Goddard. 2001. The distribution of the effects of genes affecting quantitative traits in livestock. Genet. Sel. Evol. (accepted).

Lande, R. 1981. The minimum number of genes contributing to quantitative variation between and within populations. Genetics 99:541-553.

Lande, R., and R. Thompson. 1990. Efficiency of marker assisted selection in the improvement of quantitative traits. Genetics 124:743-756

Mackinnon, M. J., and M. D. Georges. 1998. Marker-assisted preselection of young dairy sires prior to progeny-testing. Livest. Prod. Sci. 54:229-250.

Meuwissen, T. H. E., and M. E. Goddard. 1996. The use of marker haplotypes in animal breeding schemes. 28:161-176.

Meuwissen, T. H. E., and J. A. M. Van Arendonk. 1992. Potential improvement in rate of genetic gain from marker assisted selection in dairy cattle breeding schemes. J. Dairy Sci. 75:16511659.

Moreau L., A. Charcosset, F. Hospital, and A. Gallais. 1998. Marker assisted selection efficiency in populations of finite size. Genetics 148:1353-1365.

Ruane, J., and J. J. Colleau. 1996. Marker assisted selection for a sex-limited character in a nucleus breeding population. J. Dairy Sci. 79:1666-1678

Schulman, N. F., M. J. de Vries, and M. R. Dentine. 1999. Linkage disequilibrium in two stage marker-assisted selection. J. Anim. Breed. Genet. 116:99-110. 
Spelman, R. J. 1998. Major factors in marker assisted selection genetic response in dairy cattle populations. Proc. 6th World Cong. Genet. Appl. Livest. Prod. 26:365-368.

Spelman, R. J., and H. Bovenhuis. 1998. Genetic responses from marker assisted selection in an outbred population for differing marker bracket sizes and with two identified quantitative trait loci. Genetics 148:1389-1396.

Spelman, R. J., and D. J. Garrick. 1998. Genetic and economic responses for within-family marker assisted selection in dairy cattle breeding schemes. J. Dairy Sci. 81:2942-2950.

Spelman, R. J., and J. A. M. Van Arendonk. 1997. Effect of inaccurate parameter on genetic response to marker assisted selection in outbred populations. J. Dairy Sci. 80:3399-3410.

Stella, A., G. B. Jansen, P. J. Boettcher, J. P. Gibson, M. M. Lohuis, and G. Pagnacco. 2002. Accounting for uncertainty in QTL location in marker-assisted pre-selection of young bulls prior to progeny test. J. Anim. Breed. Genet. 119:15-24.
Villanueva, B., R. Pong Wong, B. Grundy, and J. A. Woolliams. 1999. Potential benefits from using an identified major gene in BLUP evaluation with truncation and optimal selection. Genet. Sel. Evol. 31:115-133.

Weller, J. I., Y. Kashi, and M. Soller. 1990. Power of daughter and granddaughter designs for determining linkage between marker loci and quantitative trait loci in dairy cattle. J. Dairy. 73:2525-2537.

Whittaker, J. C., R. N. Curnow, C. S. Haley, and R. Thompson. 1996. Using marker maps in marker assisted selection. Genet. Res. 66:255-265.

Zeng, Z. B. 1993. Theoretical basis for separation of multiple linked gene effects in mapping quantitative trait loci. Proc. Natl. Acad. Sci. 90:10972-10976.

Zhang, W., and C. Smith. 1993. Computer simulation of marker assisted selection utilizing linkage disequilibrium. Theor. Appl. Genet. 83:813-820. 